\title{
Comparison of Interpolation Methods in the Diagnosis of Carpal Tunnel Syndrome
}

\author{
Veysel Alcan1, Murat Zinnuroğlu², Gülçin Kaymak Karataş2, Elliot Bodofsky33
}

1Dikmen Vocational and Technical Anatolian High School, Ankara, Turkey

2Department of Physical Medicine and Rehabilitation, Gazi University School of Medicine, Ankara,

3Department of Physical Medicine and Rehabilitation, Cooper Medical School of Rowan University, Camden, New Jersey, USA

Background: Diagnosis of carpal tunnel syndrome is based on clinical symptoms, examination findings, and electrodiagnostic studies. For carpal tunnel syndrome, the most useful part is nerve conduction studies. However, nerve conduction study can result in ambiguous or false negative results, particularly for mild carpal tunnel syndrome. Increasing the number of nerve conduction study tests improves accuracy, but also increases time, cost, and discomfort. To improve accuracy without additional testing, the Terminal Latency index and Residual Latency are additional calculations using the minimum number of tests. Recently, the median sensory-ulnar motor latency difference was devised as another way to improve diagnostic accuracy for mild carpal tunnel syndrome.

Aims: Median sensory-ulnar motor latency difference, Terminal Latency index and Residual Latency were compared for diagnostic accuracy by severity.

Study Design: Diagnostic accuracy study.

Methods: A total of 657 subjects were retrospectively enrolled. The carpal tunnel syndrome group consisted of 546 subjects with carpal tunnel syndrome by nerve conduction study (all severities). The control group consisted of 121 subjects with no hand symptoms and normal nerve conduction study. All statistical analyses were performed using SAS. Means were compared using the one-way ANOVA test with the Bonferroni adjustment. Sensitivity, specificity, positive predictive value and negative predictive value were compared including Receiver Operating Characteristic curve analysis.

Results: For mild carpal tunnel syndrome, median sensory-ulnar motor latency difference showed the higher specificity and positive predictive value rates ( 0.967 and 0.957 , respectively) than Terminal Latency index ( 0.603 and 0.769 , respectively) and Residual Latency ( 0.818 and 0.858 , respectively). The area under the Receiver Operating Characteristio was highest for the median sensory-ulnar motor latency difference $(0.889)$, followed by the Residual Latency (0.829), and the Terminal Latency index last (0.762). Differences were statistically significant (median sensory-ulnar motor latency difference is the most accurate). For moderate carpal tunnel syndrome, sensitivity and specificity rates of Residual Latency (0.989 and 1.000) and Terminal Latency index (0.983 and 0.975$)$ were higher than median sensory-ulnar motor latency difference (0.866 and 0.958 ). Differences in area under the Receiver Operating Characteristic curve were not significantly significant, but median sensory-ulnar motor latency difference sensitivity was lower. For severe carpal tunnel syndrome, Residual Latency yielded 1.000 sensitivity, specificity, positive predictive value, negative predictive value and area beneath Receiver Operating Characteristic Curve. Differences in area under the Receiver Operating Characteristic curve were not significantly significant.

Conclusion: Median sensory-ulnar motor latency difference is the best calculated parameter for diagnosing mild carpal tunnel syndrome. It requires just a simple calculation and no additional testing. Residual Latency and Terminal Latency index are also useful in diagnosing mild to moderate carpal tunnel syndrome.

Keywords: Carpal tunnel syndrome, diagnosis, interpolation, nerve conduction

Address for Correspondence: Dr. Veysel Alcan, Dikmen Vocational and Technical Anatolian High School, Ankara, Turkey

Phone: +905055733693 e-mail: alcanveysel@gmail.com

Received: 02 October $2017 \quad$ Accepted: 24 May 2018 • DOI: 10.4274/balkanmedj.2018.1314

Available at www.balkanmedicaljournal.org

Cite this article as:

Alcan V, Zinnuroğlu M, Kaymak Karataş G, Bodofsky E. Comparison of Interpolation Methods in the Diagnosis of Carpal Tunnel Syndrome. Balkan Med J

${ }^{\circ}$ Copyright 2018 by Trakya University Faculty of Medicine / The Balkan Medical Journal published by Galenos Publishing House. 
Carpal Tunnel Syndrome (CTS) is a complex syndrome caused by compression of median nerve beneath the transverse carpal ligament (1). CTS is characterized by paresthesia, pain, atrophy, weakness and sensory abnormalities in the median nerve innervation (2).

Early diagnosis of CTS increases the chance of successful treatment. Diagnosis of CTS is based on clinical symptoms, physical examination findings, and electrodiagnostic (EDX) tests, primarily nerve conduction studies (NCS). Clinical tests can identify probable cases. EDX findings improve diagnosis (3). EDX tests are used to confirm the diagnosis of CTS and exclude other possible causes, including cervical radiculopathy (CRP) or peripheral neuropathy (4).

However, several studies show that routine EDX tests have limited sensitivity and specificity for mild CTS (4-9). An expensive, uncomfortable test with inaccurate results is not helpful. Therefore, additional calculations utilizing the minimum number of tests to improve accuracy are crucial. These tests have included the terminal latency index (TLI) and residual latency (RL), and studies have shown they improve CTS diagnostic accuracy $(3,10-12)$. A more recent technique, median sensory latency-ulnar motor latency difference (MSUMLD) was shown to be useful in a previous small study (13). The 3 techniques have never been directly compared. The aim of this study is to compare diagnosis accuracy of all of these methods in large study involving patients with CTS of all severities.

\section{MATERIALS AND METHODS}

\section{Study population}

This diagnostic accuracy study was conducted retrospectively (flowchart, Fig1). A total of 657 subjects were enrolled between January 2012 and December 2013. The CTS group consisted of 546 subjects with clinical symptoms and findings of CTS (e.g., numbness, tingling, paresthesia, pain or sensory deficits in the median nerve distribution, weakness of abductor pollicis brevis (APB) muscle and a positive Tinel's test), and abnormal NCS. The control group consisted of 121 subjects with clinical symptoms of CRP (neck pain but no hand symptoms) and normal NCS. Patients

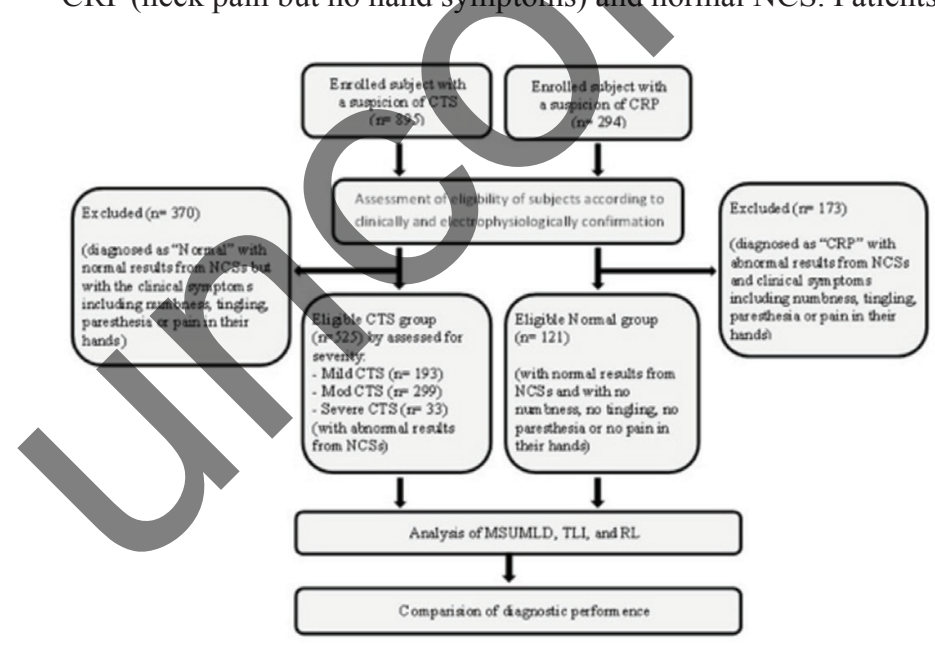

FIG. 1. Flowchart of this study. with hand symptoms and normal NCS were not included in either group. One hand of each subject was examined (14).

Exclusion criteria were a history of wrist fracture, previous median nerve surgery or injury, diabetes, chronic renal failure, gout, rheumatoid arthritis, thyroid disease, other systemic diseases associated with polyneuropathy, and plexopathy. The study protocol was approved by the school of medicine ethics committee.

\section{Nerve conduction studies}

Routine EDX tests including sensory and motor NCS for the median and ulnar nerves were performed using a Viasys Medelec Synergy EMG device. Skin temperature was maintained at 32.0 $\mathrm{C}$; room temperature between 22.0 and 25.0 C. Filter bandwidth were $20 \mathrm{~Hz}-2 \mathrm{kHz}$ for sensory NCS and $10 \mathrm{~Hz}-10 \mathrm{kHz}$ for motor NCS. Sweep speed was $1 \mathrm{msecs} /$ division for sensory NCS and 5 msecs/division for motor NCS. Sensitivity was $20 \mu \mathrm{V} /$ division for both types of NCS and increased if needed. Cup electrodes $(\mathrm{AgCl})$ with $8 \mathrm{~mm}$ in diameter were used. Distance between the recording electrodes wás $3-5 \mathrm{~cm}$.

Distance between stimulator electrodes was $3 \mathrm{~cm}$. Stimulation intensity was 10-30 mA for sensory NCS and 10-50 mA for motor NCS. Duration was 0.1-0.2 msecs for sensory NCS and 0.1-0.5 msecs for motor NCS. Supramaximal stimulation was achieved by adjusting the duration and intensity of the stimulus.

Median sensory NCS, digit II (finger)-wrist median and palm-wrist sensory nerve conduction velocities were orthodromically recorded with surface stimulation from digit II and mid palm. Latencies of the sensory nerve action potentials were measured from onset to initial negative peak.

The median motor compound muscle action potential (CMAP) was recorded with the active recording electrode placed over the midpoint of APB muscle and reference electrode distally over the thumb. The belly-tendon principle was followed and the ground electrode was placed between the stimulating and recording electrodes. Median motor distal latency (mMDL) was measured from the stimulus onset to the initial CMAP response. Median motor nerve conduction velocity (mMNCV) was determined by dividing the distance between the stimulation points by the difference in conduction times.

For the ulnar motor CMAP, the active recording electrode was placed over the belly of the abductor digiti minimi (ADM) midway between the distal wrist crease and the base of digit $\mathrm{V}$. The reference electrode was placed on the proximal phalanx of the digit V. Electrical stimulation of the ulnar nerve was done proximal to the active recording electrode at the wrist crease just lateral to the flexor carpi ulnaris tendon. Ulnar motor distal latency was measured from the stimulus onset to the initial ADM CMAP deflection.

TLI was calculated with Equation 1 and RL with Equation 2. MSUMLD was a simple subtraction (13). TLI = terminal distance $/(\mathrm{mMNCV} \times \mathrm{mMDL})$

$\mathrm{RL}=\mathrm{mMDL}-($ distal distance $(\mathrm{mm}) / \mathrm{mMNCV})$

EDX data were compared with normal reference values and categorized by our laboratories grading system (15):

- extreme CTS (absence of motor and sensory potentials)

- severe CTS (absence of sensory response and abnormal mMDL) 
- moderate CTS (abnormal sensory conduction combined with mMDL abnormalities)

- mild CTS (abnormal median sensory conduction only)

- normal requires all findings in the normal range.

In extreme CTS, median sensory and motor latencies could not be obtained and hence not included in parameter analysis. The MSUMLD requires a median sensory response and hence could not be determined in severe cases.

\section{Statistical analysis}

All statistical analyses were performed using SAS v9.4 (SAS Institute, Cary, NC). Data were reported as means \pm standard deviation (SD). Means were compared using one-way ANOVA test with the Bonferroni adjustment. For the statistical significance, the probability level of $5 \%(p<0.05)$ was required. The sensitivity and specificity were compared using Receiver Operating Characteristic (ROC) curve analysis.

\section{RESULTS}

\section{The control and the CTS groups characteristics}

According to the standards for the reporting of diagnostic accuracy studies (STARD) (16), the characteristics of the control and CTS groups are listed in Table 1. All data except gender were normally distributed. Distribution of the data was determined to be homogeneous using one way ANOVA test with the Bonferroni adjustment. The control group was significantly younger than the CTS group $(\mathrm{p}<.05)$.

\section{Comparisons of parameters between the control and the CTS} groups

Comparisons of mean values between control and all CTS groups

\begin{tabular}{|c|c|c|c|}
\hline Group & $\begin{array}{c}\mathrm{n} \text { of } \\
\text { Subject }\end{array}$ & $\begin{array}{c}\text { Female } / \\
\text { Male }\end{array} \quad \begin{array}{c}\text { Mean } \\
\text { Age } \pm S D\end{array}$ & $\begin{array}{l}\text { Age } \\
\text { range }\end{array}$ \\
\hline Control & 121 & $44.9 \pm 10.7$ & $20-66$ \\
\hline Mild CTS & 193 & $52.1 \pm 10.5$ & $21-82$ \\
\hline Moderate CTS & 299 & $51.5 \pm 11.7$ & $17-88$ \\
\hline Severe CTS & 33 & $55.2 \pm 12.3$ & $29-78$ \\
\hline Total & & $50.6 \pm 11.5$ & $17-88$ \\
\hline
\end{tabular}

are presented in Table 2. There was a statistically significant difference in all three parameters between the control group and all CTS groups $(\mathrm{p}<.0001)$.

\section{Comparison of the sensitivity and specificity}

Fig. 2 shows the lowest 1 -specificity point corresponding to the highest sensitivity value of the cut-off ROC curve for each parameter (25). Comparing controls against all CTS patients (Table 3 ), using a cutoff value of $>0.8 \mathrm{msecs}$, the MSUMLD showed

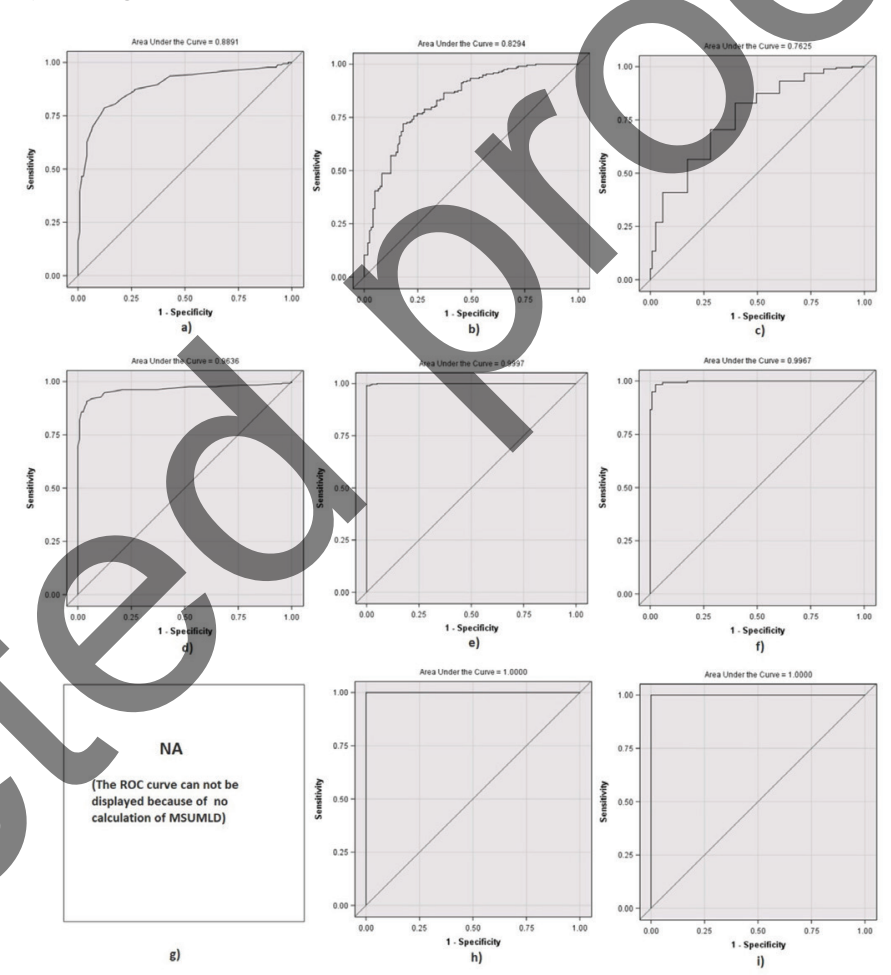

FIG. 2 a-i. The pilot of Receiver Operating Characteristic curves for each parameter a) median sensory-ulnar motor latency difference for mild carpal tunnel syndrome b) Terminal Latency index for mild carpal tunnel syndrome c) Residual Latency for mild carpal tunnel syndrome d) median sensory-ulnar motor latency difference for moderate carpal tunnel syndrome e) Terminal Latency index for moderate carpal tunnel syndrome f) Residual Latency for moderate carpal tunnel syndrome g) median sensory-ulnar motor latency difference for severe carpal tunnel syndrome h) Terminal Latency index for severe carpal tunnel syndrome, and i) Residual Latency for severe carpal tunnel syndrome.

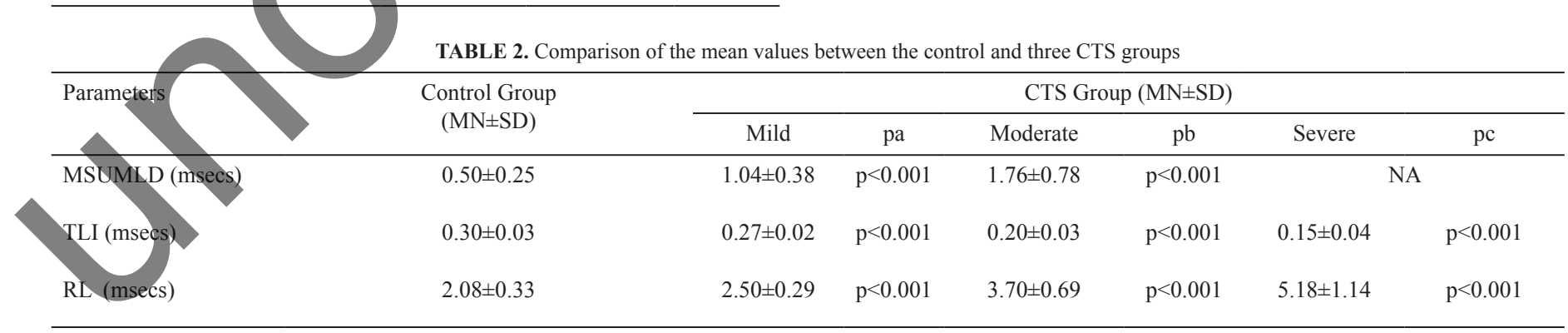

CTS: carpal tunnel syndrome; MSUMLD: median sensory-ulnar motor latency difference; TLI: terminal latency index; RL: residual latency; MN: mean value; SD: standard deviation; pa: statistical significance value of the difference between the control and mild CTS; pb: statistical significance value of difference between the control and moderate CTS; pc: statistical significance value of difference between the control and severe CTS 
sensitivity $=0.864$, specificity $=0.893$, positive predictor value $(\mathrm{PPV})=0.969$, and negative predictor value $(\mathrm{NPV})=0.632 . \mathrm{RL}>$ 2.37 msecs showed sensitivity $=0.897$, specificity $=0.818, \mathrm{PPV}=$ 0.955 , and NPV $=0.647$. For TLI $<0.26$ msecs, sensitivity $=0.729$, specificity $=0.942, \mathrm{PPV}=0.983$ and NPV $=0.496$.

Comparing the control and mild CTS groups (Table 4), cutoff values were slightly different. MSUMLD > 1.02 msecs yielded sensitivity $=0.517$, specificity $=0.967, \mathrm{PPV}=0.957$ and $\mathrm{NPV}=$ 0.582 . RL $>2.4$ msecs, sensitivity $=0.689$, specificity $=0.818$, $\mathrm{PPV}=0.858$, and NPV $=0.623$. For TLI $<0.29$ msecs, sensitivity $=$ 0.829 , specificity $=0.603, \mathrm{PPV}=0.769$, and NPV $=0.689$. The area under the ROC was highest for the MSUMLD (0.889), followed by the RL (0.829), and the TLI last (0.762), and the differences were statistically significant (MSUMLD is the most accurate).

Comparison of the control and moderate CTS is shown in Table 5. For MSUMLD $>0.95$ msecs, sensitivity $=0.866$, specificity $=0.958, \mathrm{PPV}=0.981, \mathrm{NPV}=0.743$, and area beneath the ROC Curve $=0.963$. For RL $>2.92$, sensitivity $=0.989$, specificity $=$ $1.000, \mathrm{PPV}=1.000, \mathrm{NPV}=0.975$ and area beneath the ROC Curve $=0.999$. For TLI $<0.249$, sensitivity $=0.983$, specificity $=0.975$, $\mathrm{PPV}=0.989, \mathrm{NPV}=0.959$ and area beneath $\mathrm{ROC}$ Curve $=0.996$. Differences in area under the ROC curve were not significantly significant, but MSUMLD sensitivity was lower.

Comparing controls and severe CTS (Table 6), RL > 3.39 yielded 1.000 sensitivity, specificity, PPV,

NPV and area beneath ROC Curve. For TLI $<0.21$, sensitivity $=$ 0.969 , sensitivity and PPV both $=1.000, \mathrm{NPV}=0.991$ and area beneath the ROC curve $=1.000$. Differences were not statistically significant.

TABLE 3. Comparison of the ROC parameters between the control and all CTS group

\begin{tabular}{|c|c|c|c|c|c|}
\hline \multirow[t]{2}{*}{ Parameters } & \multicolumn{5}{|c|}{ All CTS } \\
\hline & Cut-off & Sens & Spec & & $C$ \\
\hline MSUMLD (msesc) & $\geq 0.8$ & 0.864 & & & 5 \\
\hline TLI (msecs) & $\leq 0.26$ & 0.779 & & 0.496 & 0.910 \\
\hline RL (msecs) & $\geq 2.37$ & 0.897 & & 0.645 & 0.937 \\
\hline
\end{tabular}

CTS: carpal tunnel syndrome; MSUMLD: median sensory -ulnar motor latency difference; TLI: terminal latency index; RL: residual latency; Cut-off: cut-off value; Sens:sensitivity; Spec: specificity; PPV: positive predictive value; NPV=negative predictive value; AUC: area under cur

TABLE 4. Comparison of the ROC parameters between the control and mild CTS group

\begin{tabular}{lllllll}
\hline \multirow{2}{*}{\begin{tabular}{l} 
Parameters \\
\cline { 2 - 7 }
\end{tabular}} & \multicolumn{7}{c}{ Mild CTS } \\
\cline { 2 - 7 } & Cut-off & Sens & Spec & PPV & NPV & AUC \\
\hline $\begin{array}{l}\text { MSUMLD } \\
\text { (msecs) }\end{array}$ & $\geq 1.02$ & 0.517 & 0.967 & 0.957 & 0.582 & 0.889 \\
TLI (msecs) & $\leq 0.29$ & 0.829 & 0.603 & 0.769 & 0.689 & 0.762 \\
RL (msecs) & $\geq 2.4$ & 0.689 & 0.818 & 0.858 & 0.623 & 0.829
\end{tabular}

CTS: carpal tunnel syndrome; MSUMLD : median sensory -ulnar motor latency difference; TLI: terminal latency index; RL: residual latency; Cut-off: cut-off value; Sens: sensitivity; Spec: specificity; PPV: positive predictive value; NPV:negative predictive value; AUC: area under curve
TABLE 5. Comparison of the ROC parameters between the control and moderate CTS group

\begin{tabular}{|c|c|c|c|c|c|}
\hline \multirow[t]{2}{*}{ Parameters } & \multicolumn{5}{|c|}{ Mod CTS } \\
\hline & Cut-off & Sens & Spec & PPV & NPV \\
\hline $\begin{array}{l}\text { MSUMLD } \\
\text { (msecs) }\end{array}$ & $\geq 0.95$ & 0.866 & 0.958 & 0.981 & 0.743 \\
\hline TLI (msecs) & $\leq 0.249$ & 0.983 & 0.975 & 0.989 & \\
\hline RL (msecs) & $\geq 2.92$ & 0.989 & 1.000 & 1.000 & \\
\hline $\begin{array}{l}\text { CTS: carpal tu } \\
\text { motor latency } \\
\text { off: cut-off val } \\
\text { NPV:negative }\end{array}$ & $\begin{array}{l}\text { ndrome; M } \\
\text { see; TLI: to } \\
\text { s: sensitivit } \\
\text { ve value; A }\end{array}$ & $\begin{array}{l}\text { noderat } \\
\text { nal late } \\
\text { pec: spe } \\
\text { area un }\end{array}$ & & & \\
\hline
\end{tabular}

TABLE 6. Comparison of the ROC parameters between the control and

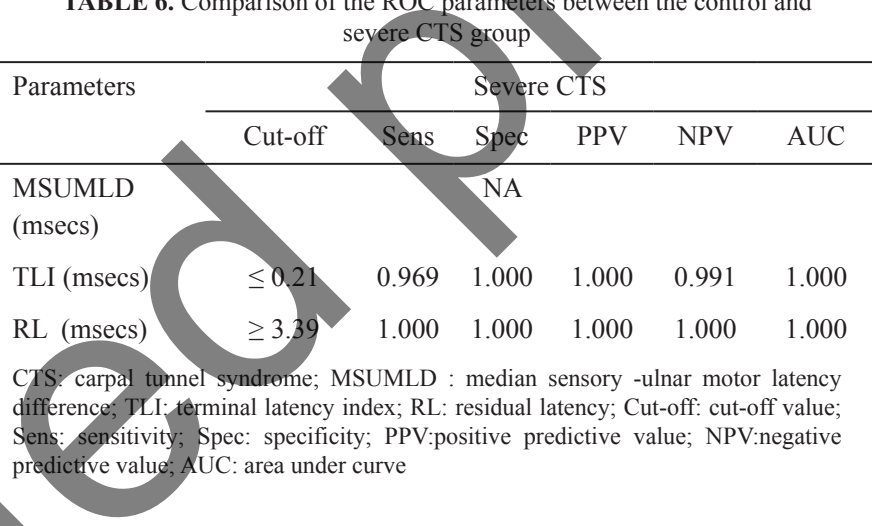

\section{DISCUSSION}

In this study, sensitivity and specificity of TLI, RL and MSUMLD were examined. In CTS, conduction abnormalities are often limited to short segments of the carpal tunnel, so normal conduction in parts of the carpal tunnel can mask slowing in mild CTS $(3,12,17)$. This lack of sensitivity, particularly for motor conduction, may result in failure to detect abnormalities $(5,18$ 21). Our results support earlier findings that sensory studies are of limited value in severe CTS because the responses are often absent $(3,22)$.

Previous studies showed a higher mean value of TLI in the control group than our study $(12,23)$. This

may be due to the population size of the control group, gender, age distribution, and differences in the normal values that each laboratory uses. Our results showed similar TLI and RL in CTS compared to other studies $(24,25)$, although one showed higher sensitivity and specificity (26). This may be due to the lack of stratification of CTS severity in some of the other studies.

MSUMLD can be a very sensitive and specific test for CTS. It is worth noting that the MSUMLD does not require mid palm stimulation, saving time and patient comfort. For MSUMLD in mild CTS, Bodofsky et al. found higher sensitivity and specificity rates than our results (13). This may be due to our larger study size, as well as the generally high sensitivity rates of other techniques in more advanced cases. All the techniques worked well for the moderate and severe cases, but they are usually not needed, as the diagnosis is straightforward in these cases. Mild CTS cases are 
hard to diagnose, and MSUMLD is the most helpful in these cases. Ulnar motor latency is usually unaffected in mild CTS, while ulnar sensory latency rises $(27,28)$. Previous studies have shown the Median and Ulnar motor latencies are significantly correlated as well as the Median and Ulnar sensory latencies in both normal and CTS, while the Median sensory and Ulnar motor are not. This can make the MSUMLD more sensitive than the (Median-Ulnar) motor or sensory latency differences.

There are some limitations to this study. For severe CTS, MSUMLD could not be compared with RL and TLI because the median sensory responses by definition could not be obtained. However, severe cases are easily diagnosed by standard criteria. There was limited information on some patients. This was a retrospective study. Diagnostic criteria were primarily EDX.

There were more female subjects in this study. However, CTS incidence is reported to be significantly higher in female population (29). Therefore, we did not need equal numbers of males and females in the control group to avoid bias. The younger control population is a limitation. Nerve conduction velocities are affected by age. There is a negative correlation between the increasing age and both NCV and amplitude per decade after the age of 20 (30). However, both median and ulnar distal latencies rise by similar degrees with increasing age, and both velocities fall to a similar degree. So a difference such as the MSUMLD should not change much with age, and this is likely also true for TLI and RL. Our normal group was referred for a clinical diagnosis of CRP and was relatively younger than the CTS group. Attempting to match the CTS group by age would have required using a much smaller normal group.

\section{CONCLUSIONS}

MSUMLD is the best calculated parameter for diagnosing mild CTS using a minimum number of tests. It requires just a simple calculation and no additional testing. RL and TLI are also useful in diagnosing mild to moderate CTS.

Conflict of interest: No conflict of interest was declared by the authors.

\section{REFERENCES}

1. Aminoff MJ.Electromyography in Clinical Practice.2thed USA:Churchill Livingstone Inc. 1987:165-196.

2. Rosenbaum R. Carpal Tunel Syndrome. In: Johnson RT, Griffin WJ: Current therapy in neurologic disease.5th ed USA: Mosby-Year book Inc. 1997:374-77.

3. Jablecki CK, Andary MT, So YT, Wilkins DE, Williams FH. Literature review of the usefulness of nerve conduction studies and electromyography for the evaluation of patients with carpal tunnel syndrome. AAEM Quality Assurance Committee. Muscle Nerve. 1993;16:1392-1414.

4. Rempel D, Evanoff B, Amadio Pe, de Krom M, Franklin G, Franzblau A, Gray R, Gerr F, Hagberg M, Hales T, Katz JN, Pransky G. Consensus criteria for the classification of carpal tunnel syndrome in epidemiologic studies. Am J Public Health.1998; 88:1447-145

5. Chang MH, Liu LH, Lee YC, Wei SJ, Chiang HL, Hsieh PF. Comparison of sensitivity of trancarpal median motor conduction velocity and conventional conduction techniques in electrodiagnosis of carpal tunnel syndrome. Clin Neurophysiol. 2006;117:984-991.

6. Keith MW, Masear V, Chung K, Maupin K, Andary M, Amadio PC,et al. Diagnosis of Carpal Tunnel Syndrome. J Am Acad Orthop Surg. 2009;17:389-396.

7. Kohara N. Clinical and Electrophysiological Findings in Carpal Tunnel Syndrome Brain Nerve. 2007;59:1229-1238
8. Sandin KJ, Asch SM, Jablecki CK, Kilmer DD, Nuckols TK. Carpal Tunnel Quality Group. Clinical quality measures for electrodiagnosis in suspected carpal tunnel syndrome. Muscle Nerve. 2010;41:444-452.

9. Demirci S, Sonel B. Comparison of sensory conduction techniques in the diagnosis of mild idiopathic carpal tunnel syndrome: which finger, which test? Rheumatol Int. 2004;24:217-220.

10. Park KM, Shin KJ, Park J, Ha SY, Kim SE. The Usefulness of Terminal Latency Index of Median Nerve and F-Wave Difference Between Median and Ulnar Nerves in Assessing the Severity of Carpal Tunnel Syndrome. J Clin Neurophysiol. 2014;31:162-168.

11. Kuntzer T. Carpal tunnel syndrome in 100 patients: sensitivity, specificity of multineurophysiological procedures and estimation of axonal loss of motor, sensory and sympathetic median nerve fibers. J Neurol Sci.1994;

12. Simovic D, Weinberg DH. The median nerve terminal latency index in carpal tunnel syndrome: a clinical case selection study. Muscle Nerve.1999; 22:573-577.

13. Bodofsky EB, Wu KD, Campellone JV, Greenberg WM, Tomaio CA. A sensitive new median- ulnar technique for diagnosing mild CarpalTunnel Syndrome. Electromyogr Clin Neurophysiol. 2005;45:139-144

14. Padua L, Pasqualetti P, Rosenbaum R. One patient, two carpal tunnels: statistical and clinical

15. Padua L, LoMonaco M, Gregori B, Valente EM, Padna R, Tonali P. Neurophysiological classification and sensitivity in 500 carpal tunnel syndrome hands. Acta Neurol Scand. 1997;96:2117.

16. Cohen JF, Korevaar DA, Altman DG, Bruns DE, Gatsonis CA Hooft L, et al STARD 2015 guidelines for reporting diagnostic accuracy studies: explanation and elaboration. BMJ Open. 2016;6(11):e012799.

17. De Lean J. Transcarpal median sensory conduction: detection of latent abnormalities mild carpal tunnel syndrome. Can J Neurol Sci. 1988;15:388-393.

18. Walters RJL, Murray NMF. Transcarpal motor conduction velocity in carpal tunnel syndrome. Muscle Nerve. 2001;24:966-968.

19. Chang MH, Wei SJ, Chiang HL, Wang HM, Hsieh PF, Huang SY. Comparison of motor conduction techniques in the diagnosis of carpal tunnel syndrome. Neurology. 2002:58:1603-1607.

20. Bodofsky EB. Diagnosing mild carpal tunnel syndrome with interpolation. Electromyogr Clin Neurophysiol. 2004;44:379- 383.

21. Bahrami MH, Rayegani SM, Nouri F. Study of interpolation method in diagnosis of carpal tunnel syndrome and comparison with midpalm antideromic sensory method. Electromyogr Clin Neurophysiol. 2009;49:259-262.

22. Boonyapisit K, Katirji B, Shapiro BE, Preston DC. Lumbrical and interossei recording in severe carpal tunnel syndrome. Muscle Nerve. 2002;25:102-105

23. Shahani BT, Young RR, Potts F, Maccabee P: Terminal latency index and late response studies in motor neuron disease, peripheral neuropathies and entrapment syndromes. Acta Neurol Scand .1979;60:118.

24. Uzar E, Tamam Y, Acar A, Yucel Y, Palancı Y, Cansever S, Ugur Cevık M, Tasdemır N. Sensitivity and specificity of terminal latency index and residual latency in the diagnosis of carpal tunnel syndrome. European Review for Medical and Pharmacological Sciences. 2011;15:1078-1084.

25. Khosrawi $\mathrm{S}$ and Dehghan F. Determination of the median nerve residual latency values in the diagnosis of carpal tunnel syndrome in comparison with other electrodiagnostic parameters. J Res Med Sci. 2013;18:934-938.

26. Perić $Z$ and Sinanović O. Sensory-Motor Index Is Useful Parameter In Electroneurographical Diagnosis Of Carpal Tunnel Syndrome. Bosnian Journal Of Basıc Medical Sciences. 2006;6 :23-27.

27. Gianneschi F, Dominici F, Milani P, Biasella A, Rossi A. Evidence of altered motor axon properties of the ulnar nerve in carpal tunnel syndrome. Clin Neurophysiol. 2007;118:1569-1576.

28. Yemisci OU, Yalbuzdag SA, Cosar SN, Oztop P, Karatas M. Ulnar nerve conduction abnormalities in carpal tunnel syndrome. Muscle Nerve. 2011;44:352-357.

29. Chammas M, Boretto J, Burmann LM, Ramos RM, Dos Santos Neto FC, Silva JB. Carpal tunnel syndrome - Part I (anatomy, physiology, etiology and diagnosis). Rev Bras Ortop 2014;49:429-36..

30. Norris AH, Shock NW, Wagman IH: Age changes in maximum conduction velocity of motor fibers of human ulnar nerves. J Appl Physiol 1953;5:589-593. 